\title{
Evidence for local mass accretion rate variations in the disc of GRS 1915+105
}

\author{
S. Migliari ${ }^{1,2}$ and T. Belloni ${ }^{2}$ \\ 1 Astronomical Institute "Anton Pannekoek" University of Amsterdam and Center for High-Energy Astrophysics, \\ Kruislaan 403, NL 1098 SJ Amsterdam, The Netherlands \\ 2 INAF - Osservatorio Astronomico di Brera, via E. Bianchi 46, 23807 Merate (LC), Italy \\ e-mail: belloni@merate.mi .astro.it
}

Received 3 February 2003 / Accepted 25 March 2003

\begin{abstract}
We have analysed 16 observations of class $\beta$ of GRS 1915+105 performed with the Rossi X-ray Timing Explorer. We show the time-resolved evolution (every $16 \mathrm{~s}$ ) of the X-ray spectral parameters during these observations. The three model independent states (A, B, C) identified in the color-color diagrams are described here in terms of the more physical (and model dependent) quantities of the disc and power law components. As in the color analysis, we observe specific and time-asymmetric transitions between states. Within a single observation, we identified a loop-like cycle of the power law spectral index that starts and ends with the ending of state $\mathrm{C}$. There is evidence of local mass accretion rate variations and mass loss during the long instability intervals (long state $\mathrm{C}$ ). This is consistent with simultaneous radio and X-ray observations that show a one-to-one relation between the long state $\mathrm{C}$ and the radio flares, that occur shortly after it: a loss of disc mass is followed by an ejection of matter in the jet.
\end{abstract}

Key words. accretion, accretion discs - black hole physics - stars: individual: GRS 1915+105 - X-rays: stars

\section{Introduction}

GRS 1915+105 was discovered in 1992 as an X-ray transient by the WATCH all sky monitor on board GRANAT (CastroTirado et al. 1992), and has been bright and active since then. In 1994, radio observations with the VLA (Very Large Array) showed radio knots moving with apparent superluminal velocities. Mirabel \& Rodríguez (1994) interpreted these expansions as collimated relativistic jet emissions with an inclination of $\sim 70^{\circ}$ from the line of sight. GRS $1915+105$, located at a distance of $\sim 11 \mathrm{kpc}$ (Fender et al. 1999), was the first galactic source to show superluminal expansion. A dynamical estimate of the mass of the compact object based on infrared observations gives $M=14 \pm 4 M_{\odot}$ and supports the idea of a black hole (Greiner et al. 2001). Since its discovery, GRS 1915+105 was extensively monitored at different wavelengths. The source shows high variability in the infrared, radio and X-ray band. Since 1996 , GRS $1915+105$ is observed at least weekly by the instruments onboard the Rossi X-ray Timing Explorer (RXTE), which confirmed its extreme X-ray variability even on very short time scale. Spectral analysis of selected observations with the Proportional Counter Array (PCA) suggested that the rapid variability of the source is caused by a thermal-viscous instability in the inner region of the accretion disc near the black hole, where the radiation pressure dominates (Belloni et al. 1997a,b).

Send offprint requests to: S. Migliari,

e-mail: migliari@astro.uva.nl
This instability causes the detected inner region of the accretion disc to disappear.

Despite the complex variability shown in the $2-30 \mathrm{keV}$ band by the PCA, it is possible to arrange all the observations from the first years of RXTE into 13 distinct classes (Belloni et al. 2000; Klein-Wolt et al. 2002). This classification is based on light curves, color-color diagrams (CD), and color-intensity diagrams, and is model-independent. Furthermore, it is possible to describe the behaviour of all 13 classes using a combination of only three source states, identified in the $\mathrm{CD}$, which Belloni et al. (2000) called state A, state $B$ and state $C$. The 2-30 keV energy spectra of GRS $1915+105$ can be described using the "standard" model for black hole candidates consisting of the superposition of a multi-temperature disc blackbody (the soft component, interpreted as an accretion disc emission) and a power law (the hard component, interpreted as derived from Comptonisation processes). These two models are obvious simplifications of the shape of the two separate components, but are helpful to characterize the spectral changes of the source on short time scales. Adopting a model-dependent approach, we can say that states A and B correspond to soft energy spectra with an observable inner region of the accretion disc, whereas state $\mathrm{C}$ is related to the "quiescent" state of Belloni et al. (1997a,b), corresponding to an unobservable inner accretion disc of variable size.

In the radio and infrared energy bands, GRS 1915+105 shows luminosity variations both on long and short time 
scales. Fender et al. (1997) observed rapid flares in the radio and infrared, and found a clear correlation between the two. They interpreted these oscillations as synchrotron emissions from repeated ejection of matter from the system. Pooley \& Fender (1997) first suggested a possible association between radio flares and X-ray dips observed on the same time scale. Mirabel et al. (1998) reported simultaneous observations in the $\mathrm{X}$-ray, radio and infrared bands, also inferring a possible relation between the IR-radio emissions and the long X-ray dip (state C). Klein-Wolt et al. (2002) established a one-to-one relation between the radio flare and the long state $\mathrm{C}$, confirming the connection of the radio jets and the disc instability in GRS 1915+105. Ueda et al. (2002) analysed a one week multiwavelength monitoring of GRS 1915+105, that covered a wide energy range from radio to gamma rays. During "plateau" observations, the radio to infrared spectra are consistent with coming from an optically thick compact jet, and constrained the infrared emitting region to a distance of $>10^{3} \mathrm{~cm}$ from the compact object. In the $\mathrm{X}$ to gamma rays spectra they found a high energy tail with no significant high energy cutoff untill $\sim 1 \mathrm{MeV}$, confirming the results of Zdziarsky et al. (2001).

In this paper we focus on the time-resolved evolution of the X-ray spectral parameters (see also Swank et al. 1998; Markwardt et al. 1999; Muno et al. 1999) during class $\beta$ observations of GRS 1915+105 (after the classification of Belloni et al. 2000). In this class, all three states are observed to last long enough to allow the accumulation of reliable spectral information from RXTE/PCA data. The paper is structured as follows. We select (Sect. 2) and analyse (Sect. 3) 16 observations of class $\beta$. We trace the behaviour and the time evolution of the spectral parameters derived from fitting the data from $\mathrm{A} / \mathrm{B} / \mathrm{C}$ states and the relations between them (Sect. 4). We discuss the results in terms of physical models (Sect. 5).

\section{Selection of observations}

As mentioned above, GRS 1915+105 shows complex and rapid variability in the X-ray band. This has been interpreted as the effect of a thermal-viscous instability of the inner region of the accretion disc (Belloni et al. 1997a,b; see also e.g. Taam et al. 1997; Nayakshin et al. 1999; Janiuk et al. 2000; Zampieri et al. 2000). Our aim is to follow the temporal evolution of the spectral parameters of the source during these instability intervals using RXTE/PCA data. For the spectral analysis we used Standard2 data, which have a good spectral resolution for our purposes. However, this choice limits the classes of observations we can analyse. The Standard 2 data have a timing resolution of $16 \mathrm{~s}$. This implies that in order to trace the evolution of the spectral parameters during the instability intervals we have to select observations in which the length of the three states is substantially longer than $16 \mathrm{~s}$. From the classification by Belloni et al. (2000), we selected 16 observations of class $\beta$. The log of the selected observations is shown in Table 1.

\section{Data analysis}

For each observation, we produced light curves at $1 \mathrm{~s}$ time resolution using Standardl data in order to identify the long
Table 1. Observation ID, RXTE orbits, duration of RXTE orbits and duration of state $\mathrm{C}$ (the upper limits indicate that the observation is interrupted while in state $\mathrm{C}$ ) of the 16 observations analysed.

\begin{tabular}{|c|c|c|c|}
\hline Obs ID & \# Orb & $\Delta T_{\text {obs }}(\mathrm{s})$ & $\Delta T$ State $\mathrm{C}(\mathrm{s})$ \\
\hline \multirow[t]{2}{*}{$10408-01-10-00$} & $\mathrm{a}$ & 3480 & 432 \\
\hline & $\mathrm{b}$ & 3397 & 448 \\
\hline \multirow[t]{6}{*}{ 20186-03-03-01 } & $\mathrm{a}$ & 1792 & \\
\hline & $\mathrm{b}$ & 2197 & 704 \\
\hline & $\mathrm{c}$ & 2501 & 688 \\
\hline & $\mathrm{d}$ & 2933 & 560 \\
\hline & & & 592 \\
\hline & $\mathrm{e}$ & 256 & - \\
\hline \multirow[t]{2}{*}{$20402-01-43-00$} & $\mathrm{a}$ & 9199 & 528 \\
\hline & & & 560 \\
\hline \multirow[t]{4}{*}{$20402-01-44-00$} & $\bar{a}$ & 9288 & $>560$ \\
\hline & & & 688 \\
\hline & & & 672 \\
\hline & & & 704 \\
\hline \multirow{5}{*}{$20402-01-45-00$} & $\bar{a}$ & 2516 & 544 \\
\hline & & & 528 \\
\hline & $\mathrm{b}$ & 2267 & - \\
\hline & $\mathrm{c}$ & 4480 & 528 \\
\hline & $\mathrm{d}$ & 2025 & - \\
\hline \multirow{5}{*}{$20402-01-45-03$} & $\bar{a}$ & 3072 & 528 \\
\hline & $\mathrm{b}$ & 3313 & 512 \\
\hline & $\mathrm{c}$ & 3425 & 720 \\
\hline & & & 656 \\
\hline & $\mathrm{d}$ & 276 & - \\
\hline \multirow[t]{8}{*}{ 20402-01-46-00 } & $\mathrm{a}$ & 383 & - \\
\hline & $\mathrm{b}$ & 3407 & 592 \\
\hline & & & 624 \\
\hline & $\mathrm{c}$ & 3167 & 592 \\
\hline & & & 640 \\
\hline & & & $>448$ \\
\hline & $\mathrm{d}$ & 2797 & 528 \\
\hline & & & 656 \\
\hline \multirow[t]{2}{*}{$20402-01-52-01$} & $\mathrm{a}$ & 2057 & 736 \\
\hline & & & $>480$ \\
\hline $20402-01-52-02$ & $\bar{a}$ & 2024 & 768 \\
\hline \multirow[t]{3}{*}{$20402-01-53-00$} & $\mathrm{a}$ & 8448 & 480 \\
\hline & & & 464 \\
\hline & $\mathrm{b}$ & 3477 & 512 \\
\hline \multirow{3}{*}{$20402-01-59-00$} & $\bar{a}$ & 3367 & 544 \\
\hline & $\mathrm{b}$ & 3399 & 528 \\
\hline & $\mathrm{c}$ & 2983 & - \\
\hline \multirow[t]{4}{*}{$30182-01-03-00$} & $\bar{a}$ & 1024 & - \\
\hline & $\mathrm{b}$ & 4736 & $>704$ \\
\hline & $\mathrm{c}$ & 5248 & - \\
\hline & $\mathrm{d}$ & 11612 & - \\
\hline \multirow[t]{2}{*}{$40115-01-04-00$} & $\bar{a}$ & 3254 & 896 \\
\hline & & & 880 \\
\hline \multirow[t]{4}{*}{$40703-01-18-00$} & $\mathrm{a}$ & 8413 & $>496$ \\
\hline & & & 992 \\
\hline & & & $>464$ \\
\hline & & & 880 \\
\hline 40703-01-35-00 & $\mathrm{a}$ & 1779 & 688 \\
\hline \multirow{2}{*}{$40703-01-35-01$} & $\mathrm{a}$ & 3935 & 816 \\
\hline & $\mathrm{b}$ & 4096 & $>640$ \\
\hline
\end{tabular}


$\mathrm{A} / \mathrm{B} / \mathrm{C}$ intervals and separate them clearly from faster oscillations. For each RXTE orbit, we extracted energy spectra in the range 3-25 keV, with an integration time of $16 \mathrm{~s}$ (from Standard2 data), We created the detector response matrices with pcarsp v7.10, and to each spectrum we subtracted the background, estimated using pcabackest v.2.1e. In our spectral analysis, we considered the "standard" model for BHCs which consists in the superposition of two spectral components: a disc-blackbody and a power law. Our choice of a simple power law instead of a more accurate Comptonising model is motivated by the need of a simple model for efficiently performing a large number of fits with automatic procedures. In order to check that this model yields meaningful parameters in the framework of Comptonising models, we fitted a few spectra for each observation with the bmc model in XSPEC. These fits give values of the spectral index $\Gamma$ consistent with those of a simple power law model, with a small systematic deviation to larger values of $\sim 3 \%$, and comparable distribution of fluxes between the soft and the hard component. It is important to stress that the disc-blackbody model does not consider relativistic effects and, as we are investigating the inner region of the disc close to the compact object, this is an approximation that we have to treat with caution for a more detailed study of the physics involved. From the normalization of the thermal component we are able to calculate the inner radius of the accretion disc $\left(R_{\text {in }}=D \sqrt{(N / \cos \theta)} \mathrm{km}, D\right.$ is the distance of the source in units of $10 \mathrm{kpc}, \theta$ is the inclination angle of the disc, and $N$ is the normalization of the disc-blackbody). However, this radius is always underestimated, since what we calculate is not the effective radius of the disc (the radius corresponding to the higher inner temperature), but the radius derived from the color temperature (see Merloni et al. 1999). All the energy spectra are fitted using this model, modified with photoelectric absorption (the equivalent hydrogen column density was fixed to $7 \times 10^{22} \mathrm{~cm}^{-2}$, see Sect. 3.1) and adding a Gaussian emission line with a fixed central energy which takes into account an excess around $6.4 \mathrm{keV}$. We added a $1 \%$ systematic error, the standard estimated residual error on the calibration. The value of the reduced $\chi^{2}$ was usually around 1 , although for some fits it was slightly higher. All extractions and fits were performed with automatic procedures.

\subsection{Column density}

Photoelectric absorption modifies the emission spectrum at low energies in the PCA band, where the disc component dominates the flux. The value of the equivalent hydrogen column density $N_{\mathrm{H}}$ used to calculate the absorption influences the normalization of the disc-blackbody component, and thus the value of the inner radius $R_{\text {in }}$. These two quantities $\left(N_{\mathrm{H}}\right.$ and $\left.R_{\text {in }}\right)$ are therefore strongly coupled when fitting the spectra. In order to trace the variability in the derived inner radius, we had to fix the $N_{\mathrm{H}}$ value (we realistically suppose that the column density does not change within time scales of the order of half an hour). In order to find the best $N_{\mathrm{H}}$ value, we have analysed several class $\chi_{1}$ and $\chi_{3}$ observations (in the classification of Belloni et al. 2000). The advantage in these classes of
Table 2. Results from the spectral analysis of 15 spectra of class $\chi$ observations. It is shown the observation ID, the number of the orbit and the best-fit column density value $\left(N_{\mathrm{H}}\right)$.

\begin{tabular}{lll}
\hline \hline Obs ID & Orbit & $N_{\mathrm{H}}\left(\times 10^{22} \mathrm{~cm}^{-2}\right)$ \\
\hline $10408-01-23-00$ & $\mathrm{a}$ & $7.22 \pm 0.05$ \\
& $\mathrm{~b}$ & $7.28 \pm 0.14$ \\
& $\mathrm{c}$ & $7.61 \pm 0.11$ \\
\hline $10408-01-24-00$ & $\mathrm{a}$ & $6.37 \pm 0.12$ \\
& $\mathrm{~b}$ & $6.40 \pm 0.13$ \\
& $\mathrm{c}$ & $6.72 \pm 0.10$ \\
\hline $10408-01-27-00$ & $\mathrm{a}$ & $7.13 \pm 0.13$ \\
& $\mathrm{~b}$ & $7.23 \pm 0.16$ \\
\hline $20402-01-52-00$ & $\mathrm{a}$ & $5.76 \pm 0.14$ \\
& $\mathrm{~b}$ & $5.70 \pm 0.12$ \\
& $\mathrm{c}$ & $5.78 \pm 0.10$ \\
\hline $30402-01-09-00$ & $\mathrm{a}$ & $5.78 \pm 0.14$ \\
& $\mathrm{~b}$ & $5.96 \pm 0.13$ \\
\hline $30703-01-16-00$ & $\mathrm{a}$ & $5.65 \pm 0.15$ \\
& $\mathrm{~b}$ & $5.74 \pm 0.16$ \\
\hline
\end{tabular}

observations is that the soft (disc) component is not detectable in the PCA energy range and therefore there are no variations in the soft spectral component that can influence the estimated $N_{\mathrm{H}}$ value. Because of this characteristic and the absence of significant flux variability over each observation, we can accumulate the spectra over a whole RXTE orbit to obtain better statistics. The observations we have analysed and the best-fit $N_{\mathrm{H}}$ values are shown in Table 2. For the different observations, the column density varies within the range $5.7-7.6 \times 10^{22} \mathrm{~cm}^{-2}$, consistent with values found by other authors (Ebisawa et al. 1995; Belloni et al. 1997b; Muno et al. 1999). We have analysed the energy spectra of state $\mathrm{C}$ intervals for several class $\beta$ observations of GRS $1915+105$, fixing the $N_{\mathrm{H}}$ at different values in the range we found for class $\chi$. We found a general improvement of the $\chi^{2}$ in the estimation of the spectral parameters using $N_{\mathrm{H}}=7 \times 10^{22} \mathrm{~cm}^{-2}$. The column density was therefore fixed to $7 \times 10^{22} \mathrm{~cm}^{-2}$ for all observations discussed below. We found however that the results remain qualitatively the same compared to $N_{\mathrm{H}}=6 \times 10^{22} \mathrm{~cm}^{-2}$.

\section{Results}

For each RXTE orbit within our observations, we isolated all $\mathrm{A} / \mathrm{B} / \mathrm{C}$ intervals long enough to obtain significant results using spectra accumulated over $16 \mathrm{~s}$, but also analysed the whole orbit (including faster oscillations for completeness). Of course the results from the oscillating parts, having been sampled at $16 \mathrm{~s}$, are not covered in an optimal way, but we concentrate our analysis on the slower variations. The fitting procedure resulted in very good values of the reduced $\chi^{2}$ for almost all the spectra (usually $\sim 1$ ), with the exception of very few spectra, usually during state B.

In all observations, the light curves during the instability show the same characteristics: a hard state $\mathrm{C}$, followed by an isolated spike at the end of the state $\mathrm{C}$, a shorter soft low-flux 


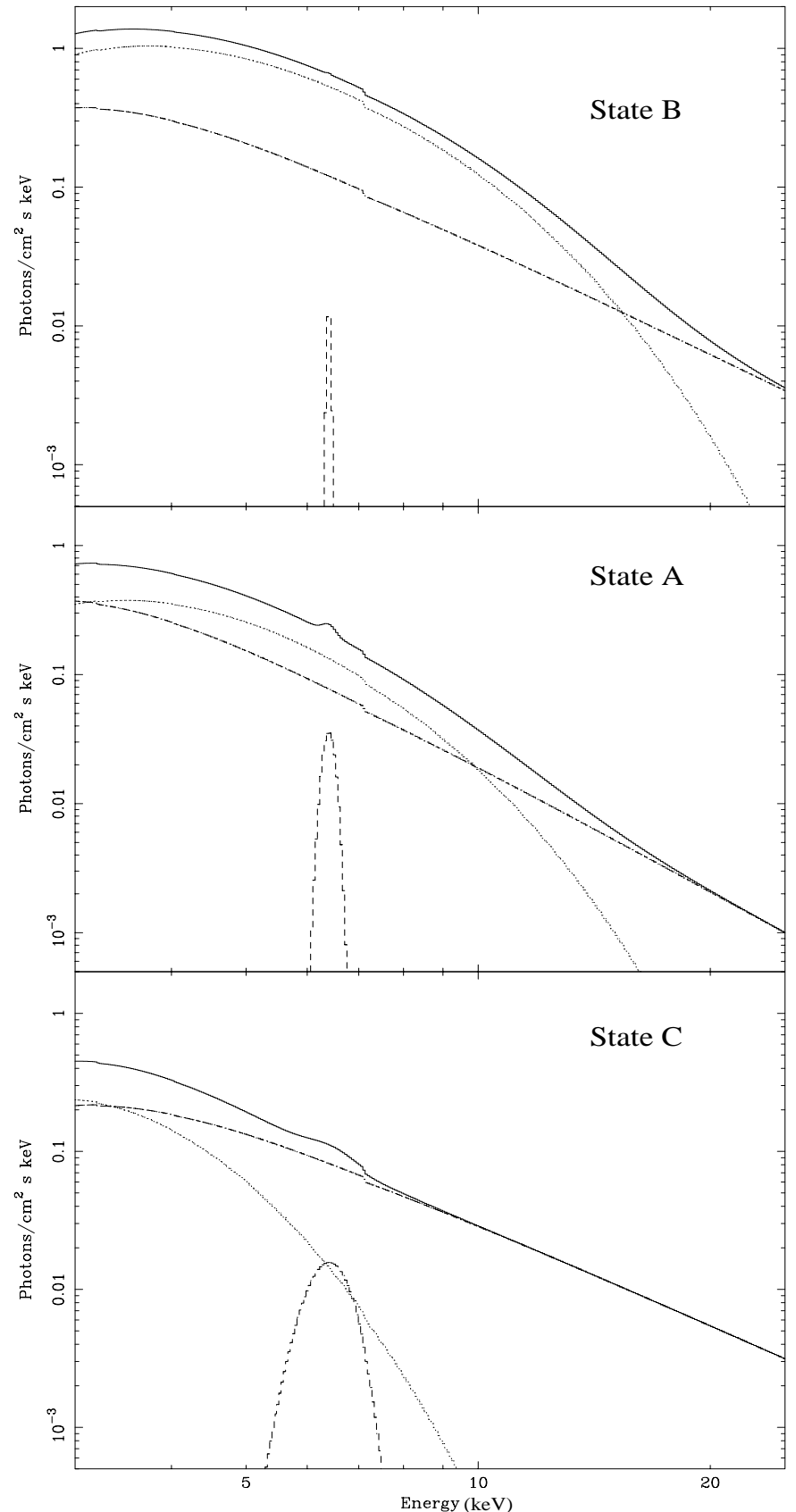

Fig. 1. Examples of fitting spectral models for the three states A/B/C. The model (solid line) is the superposition of a power law (dasheddotted), a disc blackbody (dotted) and a Gaussian emission line (dashed).

state A shifting slowly into state B (see e.g. Swank et al. 1998; Markwardt et al. 1998; Mirabel et al. 1998; Belloni et al. 2000; Klein-Wolt et al. 2002). In Fig. 1 we show three representative model spectra for the three states. Notice the difference in relative contribution of the two main components. As an example, in Fig. 2 we show the evolution of the spectral parameters for the whole first orbit of observation 20402-01-44-00 (for clarity, we show typical $90 \%$ error bars only for a few points). In the first panel from the top we show the $2-60 \mathrm{keV}$ light curve, in the second the power law spectral index, in the third the inner temperature (in $\mathrm{keV}$ ) of the accretion disc, in the fourth the inner radius of the disc (in $\mathrm{km}$ ), and in the fifth and sixth panels we show the 3-25 keV luminosity of the hard power law component and the bolometric luminosity of the disc blackbody component as calculated from the best-fit parameters (normalisation and $\Gamma$ from the fitting power law, and $R_{\text {in }}$ and $T_{\text {in }}$ from the fitting disc blackbody model) using a distance of $11 \mathrm{kpc}$ and an inclination of the disc of $66^{\circ}$ (Fender et al. 1999) thought to be perpendicular to the radio jet (see also Maccarone 2002). The corresponding correlations between parameters are shown in Fig. 2. Here, the results for the oscillating parts are included, as they give a general idea of the spectral changes during faster events.

The time evolution of the different parameters can be summarised as follows.

- power law parameters: From Fig. 2, we can see that the evolution of the power law spectral index $\Gamma$ shows a "cycle" which starts and ends at the end of a state $\mathrm{C}$ interval. Following the first cycle, starting $\sim 800 \mathrm{~s}$ from the beginning of the observation, we notice that during state $\mathrm{C} \Gamma$ follows the light curve, starting from $\sim 2.8$ at the beginning, decreasing to 2.4 , then increasing again to reach it maximum $(\sim 3.3)$ at the end of state $C$, in correspondence with the "spike" in the light curve. Then, during the state A and B intervals, it gradually decreases until it reaches a value of about 3. After this, the faster oscillations start and $\Gamma$ oscillates in the range $2.8-3.3$. Notice that $\Gamma$ correlates with the count rate during state $\mathrm{C}$, but not during state $\mathrm{B}$. The power law flux (fifth panel from the top) shows large variations during state $\mathrm{C}$, and a much less variable behaviour during states A/B.

- Disc parameters: In the third and fourth panels from the top of Fig. 2, we show the evolution of the accretion disc parameters (inner temperature and radius). It is evident the confirmation of the results obtained by Belloni et al. (1997b): during state C, the inner radius $R_{\text {in }}$ increases whereas the inner temperature $k T_{\text {in }}$ decreases, both rather fast. This is compatible with the idea of a "missing", or simply unobservable, inner region of the disc. At the end of state $\mathrm{C}$, the inner radius goes down to a minimum around $30 \mathrm{~km}$ corresponding to state A, without a strong jump at the "spike". Moreover, the inner disc radius does not change much during state $\mathrm{A}$ and $\mathrm{B}$, remaining at its minimum value, and even during the oscillations its variations are rather limited. The inner disc temperature, on the other hand, remains low $\sim 0.8 \mathrm{keV}$ and constant for much of state $\mathrm{C}$, then towards the end starts increasing. This increase continues through states $\mathrm{A}$ and $\mathrm{B}$ up to $\sim 2 \mathrm{keV}$, again without dramatic changes in correspondence with the "spike". The "spike" is essentially caused by a sharp transition in the properties of the power law component. The disc flux evolution (sixth panel from the top) shows a constant flux throughout state $\mathrm{C}$, with some evidence of the "spike", and an increased flux during states $\mathrm{A} / \mathrm{B}$ and during the oscillations. Notice that over the whole orbit the disc flux dominates over the power law flux.

In Fig. 3, we show the correlations between the best-fit parameters: inner disc temperature, inner disc radius and power law 


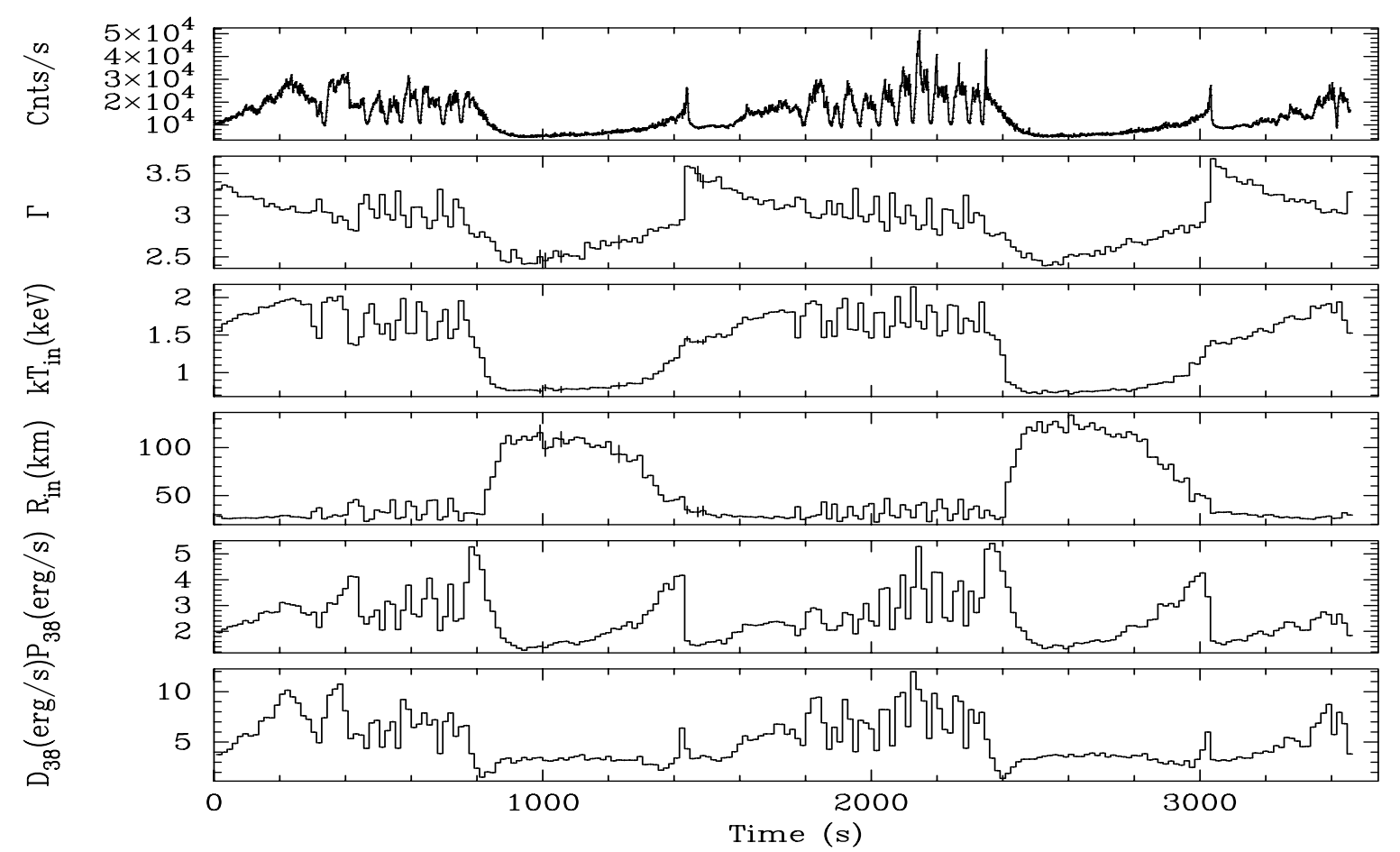

Fig. 2. Time-resolved evolution of the spectral parameters from the analysis of an RXTE orbit of the observation 20402-01-44-00. From top to bottom: 2-60 keV light curve (5 PCUs); power law spectral index $\Gamma$; inner disc temperature $\left(k T_{\text {in }}\right.$ in $\left.\mathrm{keV}\right)$; inner disc radius $\left(R_{\text {in }}\right.$ in $\left.\mathrm{km}\right)$; 3-25 keV luminosity of the power law component $P_{38}$ in units of $10^{38} \mathrm{erg} / \mathrm{s}$; bolometric luminosity of the disc component $D_{38}$ (in units of $10^{38} \mathrm{erg} / \mathrm{s}$ ) calculated using a distance of $11 \mathrm{kpc}$ and a disc inclination of $66^{\circ}$ (Fender et al. 1999). Typical $90 \%$ error bars are shown for a few points.

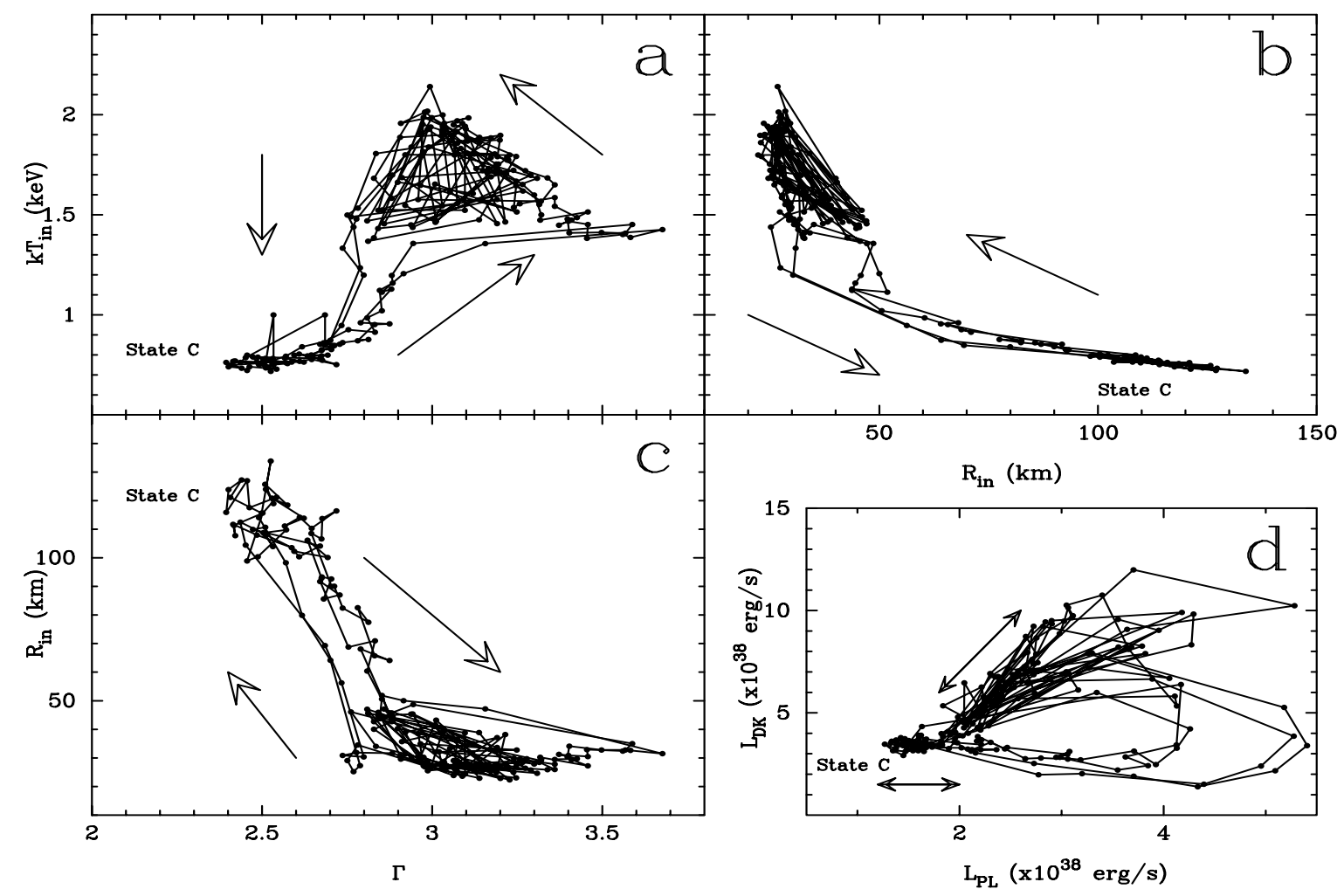

Fig. 3. Correlations between the spectral parameters of Fig. 2. Panel a) power law spectral index $\Gamma$ vs. inner disc temperature; panel b) inner disc temperature vs. inner disc radius; panel c) inner disc radius vs. $\Gamma$; panel d) luminosity of the disc component vs. luminosity of the power law component. 


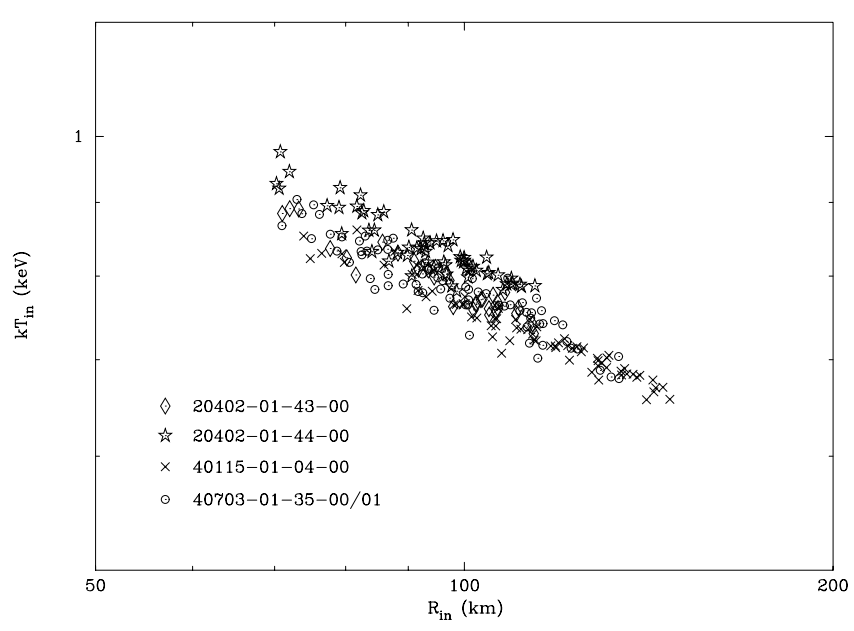

Fig. 4. Inner disc temperature as a function of inner radius for five GRS 1915+105 observations. We isolated the intervals of lower count rate of state $\mathrm{C}$.

index, plus the disc/power law flux correlation. Here, obvious and complex correlations are apparent. This behaviour is common to all observations in our sample. In particular, it is clear that the transitions between state $\mathrm{C}$ and the softer states is not time-reversible. This is evident from the "loop-like" shape of the observed correlations (see also Vilhu \& Nevalainen 1998).

Here we concentrate on the correlation between inner disc radius and temperature (panels labeled "b"), as this plot gives some indication of the local accretion rate through the disc at the measured inner radius. Despite the uncertainties on the absolute values of radius measurements mentioned above (and caused also by uncertaintes on the distance to the source), relative values can be useful in determining what happens to the disc during the instability intervals. From Fig. 3b, one can see that during state $\mathrm{C}$ there is a clear inverse correlation between inner radius and temperature, correlation which is rather similar throughout the whole of state $\mathrm{C}$. However, while entering and leaving state $\mathrm{C}$, the source follows rather different paths, mostly due to the presence of the "spike" at the end of state C. As usual, faster oscillations are not optimally sampled and result in a noisy loop structure. Vilhu \& Nevalainen (1998) found a ring-shaped pattern in the $\mathrm{CD}$ during fast quasi-regular variations in the light curve count rate (class $\rho$ observations), where the source is rapidly oscillating between state $\mathrm{C}$ and state $\mathrm{B}$. We notice that a loop (ring-like) structure can be seen also in Fig. 3d (power law luminosity $L_{\mathrm{pl}}$ vs. disk blackbody luminosity $L_{\mathrm{dk}}$ ), where the transitions between the hard state $\mathrm{C}$ and the soft states $\mathrm{A} / \mathrm{B}$ have a ring shaped pattern. The difference from the fast variations seen in class $\rho$ observations seems to be only the duration of the single states. In $\beta$ observations the source lies for a longer time in each state $(\mathrm{A} / \mathrm{B} / \mathrm{C})$ where the power law and the disk blackbody luminosities form two elongated branches. Faster ring-like transitions happen between these two branches. This suggests that the physical processes that cause state transitions are the same independently on the time that the source spends in one state (seconds as in class $\rho$ or tens of minutes as in class $\beta$ ).

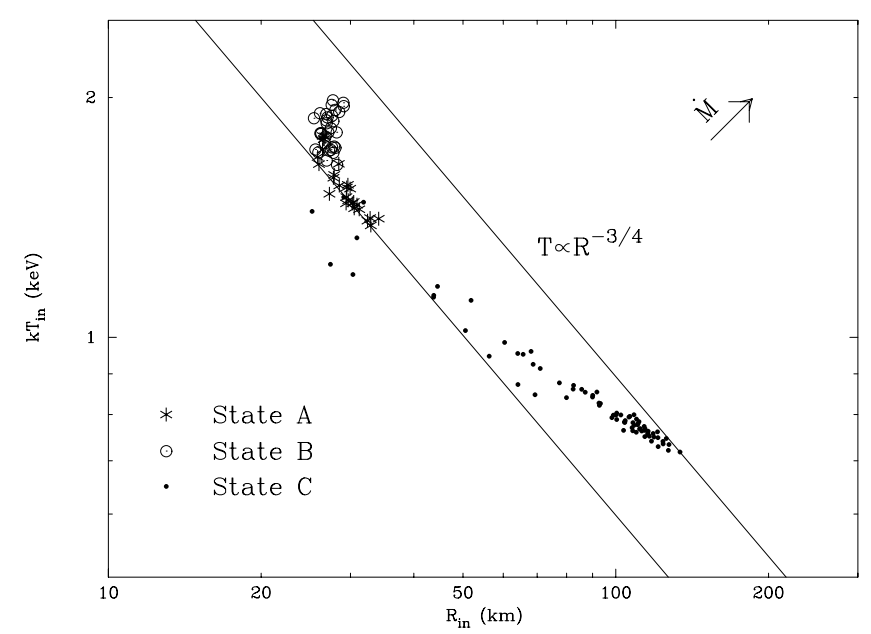

Fig. 5. Inner disc temperature versus inner disc radius. We plot the points corresponding to two intervals of instability of the observation 20402-01-44-00: state C, state A and the beginning of state B (without the rapid flares; see Fig. $2 b$ ). We excluded $\sim 50 \mathrm{~s}$ of data around the spikes.

\section{Discussion}

The evolution in the $R_{\text {in }}-k T$ plane can be read in terms of variations in the local mass accretion rate flowing through the inner radius of the optically thick part of the accretion flow. In Fig. 4, we have isolated the interval corresponding to the lower count rate of state $\mathrm{C}$ for five observations. We notice that all the points of the different observations follow the same correlation, that can be fitted with a power law with slope $\sim-0.4$. At constant accretion rate, from a standard Shakura \& Sunyaev (1974) disc, a slope -0.75 would be expected. This indicates that there are systematic variations in the local disc mass accretion rate during state $\mathrm{C}$, where $\dot{M}$ is at a relative maximum at the bottom of state $\mathrm{C}$, when the inner disc radius is larger. In order to follow the parameter evolution throughout states A and B, in Fig. 5 we plot the points from two instability intervals of the observation 20402-01-44-00. Here two lines mark the relation $T_{\text {in }} \propto R_{\text {in }}^{-3 / 4}$ for two values of the accretion rate (increasing to the upper right). The correlation shown in Fig. 5 is evident in the bottom part of the plot. However, state A points, when the inner radius is around $35 \mathrm{~km}$, follow precisely the expected -0.75 slope, indicating that the local accretion rate remains constant during state $\mathrm{A}$. Then, when state $\mathrm{B}$ is reached, the inner radius reaches its minimum value, probably corresponding to the innermost stable orbit around the black hole, while the temperature continues to grow, leading to a further increase of the disc accretion rate.

This overall behaviour indicates that there is a loss of matter in the inner region of the disc during the instability (state $\mathrm{C}$ ), with the local disc accretion rate oscillating between two values during the instability (the difference in local mass accretion rate between these two values is of a few $\left.10^{-8} M_{\odot} / \mathrm{yr}\right)$. This is consistent with the results by Klein-Wolt et al. (2002), who find a one-to-one relation between long state $\mathrm{C}$ intervals and radio flares, and that these outflow emissions occur shortly after the instability intervals, where we found a loss of matter in the disc. 
Finally, we notice that the minimum inner radius of $\sim 30 \mathrm{~km}$ reached during state $\mathrm{B}$ appears to be the same in all observations in our sample, consistent with its association with the minimum stable orbit around the black hole.

\section{Conclusions}

We have analysed 16 class $\beta$ PCA/RXTE observations of GRS $1915+105$, and studied the time-resolved evolution of the spectral parameters. We can summarise our results as follows:

- The three states (A, B, C) identified in the CD (Belloni et al. 2000) are described here in terms of the more physical quantities of the disc and power law components. As in the color analysis, we observe specific and timeasymmetric transitions between states. These transitions, already noticed in Belloni et al. (2000), are now translated into more physical (and model dependent) parameter evolutions. A large degree of "clustering" of spectral and timing parameters in a few states were already noticed by Markwardt et al. (1998) studying one class $\beta$ observation. A loop-like structure is found and a ring-shaped pattern as found by Vilhu \& Nevalainen (1998) in (fast flux oscillating) class $\rho$ observations can be seen also in $\beta$ observations (Fig. 3d). The only difference with the class $\rho$ seems to be the presence of two "branches" that are formed because of the longer duration of the hard $(\mathrm{C})$ and the $\operatorname{soft}(\mathrm{A} / \mathrm{B})$ states.

- Within a single observation, the power law spectral index $\Gamma$ has a cycle that starts and ends with the ending of state $\mathrm{C}$ (i.e. in correspondence with the spike).

- There is evidence, consistent with simultaneous radio and X-ray observations (see Klein-Wolt et al. 2002), of local mass accretion rate variations and mass loss during the long instability intervals (long state C).

Acknowledgements. TB thanks the Cariplo Foundation for financial support. We would like to thank Rob Fender for comments on a draft of this manuscript.

\section{References}

Belloni, T., Klein-Wolt, M., Méndez, M., van der Klis, M., \& van Paradijs, J. 2000, A\&A, 355, 271

Belloni, T., Méndez, M., King, A. R., van der Klis, M., \& van Paradijs, J. 1997a, ApJ, 479, L145

Belloni, T., Méndez, M., King, A. R., van der Klis, M., \& van Paradijs, J. 1997b, ApJ, 488, L109

Bradt, H. V., Rothschild, R. E., \& Swank, J. H. 1993, A\&AS, 97, 355-360

Castro-Tirado, A. J., Brandt, S., \& Lund, S. 1992, IAU Circ., 5590

Ebisawa, K., White, N. E., Kotani, T., \& Harmon, A. 1995, IAU Circ., 6171, 1

Fender, R. P., Pooley, G. G., Brocksopp, C., \& Newell, S. J. 1997, MNRAS, 290, L65

Fender, R. P., Garrington, S. T., McKay, D. J., et al. 1999, MNRAS, 304, 865

Greiner, J., Cuby, J. G., \& McCaughrean, M. J. 2001, Nature, 414, 522

Janiuk, A., Czerny, B., \& Siemiginowska, A. 2000, 542, L33

Klein-Wolt, M., Fender, R. P., Pooley, G. G., et al. 2002, MNRAS, 331,745

Maccarone, T. J. 2002, MNRAS, 336, 1371

Markwardt, C. B., Swank, J. H., \& Taam, R. E. 1999, ApJ, 513, L37

Merloni, A., Fabian, A. C., \& Ross, R. R. 1999, MNRAS, 313, 193-197

Mirabel, I. F., \& Rodríguez, L. F. 1994, Nature, 371, 46

Mirabel, I. F., Dhawan, V., Chaty, S., et al. 1998, A\&A, 330, L9

Muno, M. P., Morgan, E. H., \& Remillard, R. A. 1999, ApJ, 527, 321

Nayakshin, S., Rappaport, S., \& Melia, F. 2000, ApJ, 535, 798

Pooley, G. G., \& Fender, R. P. 1997, MNRAS, 292, 925

Swank, J., Chen, X., Markwardt, C., \& Taam, R. 1998, Proc. of the Conf., Accretion Processes in Astrophysics: Some Like it Hot, held at U. Md., October 1997, ed. S. Holt, \& T. Kallman

Taam, R. E., Chen, X., \& Swank, J. H. 1997, ApJ, 485, L83

Ueda, Y., Yamaoka, K., Sánchez-Fernández, C., et al. 2002, ApJ, 571, 918

Vilhu, O., \& Nevalainen, J., ApJ, 508, L85

Zampieri, L., Turolla, R., \& Szuszkiewicz, E. 2001, MNRAS, 325, 1266

Zdziarsky, A. A., Grove, J. E., Poutanen, J., Rao, A. R., \& Vadawale, S. V. 2001, ApJ, 554, L45 